\title{
Del MOOC al SPOC. Una experiencia para el aprendizaje líquido de la Química básica
}

\author{
M $^{\mathrm{a}}$ Teresa Pardo ${ }^{\mathrm{a}}$, Isabel Morera ${ }^{\mathrm{a}}$, Juan A. Llorens-Molina ${ }^{\mathrm{a}}$, Carmen Gómez \\ e Ignacio Despujol ${ }^{b}$
}

tpardo@qim.upv.es, imorera@upvnet.upv.es, juallom2@qim.upv.es, mcgomez@qim.upv.es, ndespujol@asic.upv.es

aDepartamento de Química

bDepartamento de Ingeniería de la Construcción y de Proyectos de Ingeniería Civil

Universitat Politècnica de València.

Camino de Vera s/n. 46071-València

\begin{abstract}
The introduction of Information and Communication Technologies (ICT) means an undeniable and irreversible change of educational paradigm offering alternatives for attending teaching which are reflected in teachinglearning process. The teacher should compete with many technologies presenting different options for students' formation. The open and participative courses favor the relationship and make feasible the communication. The Higher Education requires new models which allow training people able to adapt and survive in changing environments. From this point of view, it is said of liquid education or learning, appropriate to our times. It is based on the use of technologies and the adaptation of knowledge to people. It is about an education according to circumstances, which is adapted to context and virtual behavior of people. This communication shows a model of liquid learning for basic chemistry, an SPOC (Small Private On line Course) aimed at a more reduced public than the MOOCs. It is progressive and personalized, with instantaneous feedback. The reasons leading teachers to design this sort of initiative have been described, the same way that the content analysis and the authors' workload.
\end{abstract}

Keywords:

Chemistry, liquid learning, platform UPV[X], MOOC, SPOC 


\begin{abstract}
Resumen
El cambio del paradigma educativo que ha supuesto la introducción de las TIC es innegable e irreversible ofreciendo alternativas a la docencia presencial que se ven reflejadas en el proceso de enseñanza-aprendizaje. El profesor debe competir con tecnologías varias que proporcionan diferentes opciones de formación a sus estudiantes. Los cursos abiertos y participativos favorecen la relación y posibilitan la comunicación. La Educación Superior requiere de nuevos modelos que permitan la formación de personas con capacidad de adaptarse y de sobrevivir en un ambiente cambiante. Desde esta perspectiva se habla de educación o aprendizaje líquido, acorde a nuestros tiempos, basado en el uso de tecnologías, adaptando el conocimiento a las personas. Se trata de una educación a medida de las circunstancias, que se ajusta al contexto y al comportamiento virtual de las personas. Esta comunicación presenta un modelo de aprendizaje líquido de la Química básica, un curso SPOC "Small Private Online Courses", dirigido a un público más reducido que los MOOC, progresivo, personalizado, con retroalimentación instantánea. Se describen los motivos que han llevado a los profesores a diseñar este tipo de iniciativa, el análisis de los contenidos y el volumen de trabajo que ha supuesto para los autores.
\end{abstract}

\title{
Palabras clave:
}

Química, aprendizaje líquido, plataforma UPV[X], MOOC, SPOC

\section{Introducción}

Con la incorporación al Espacio Europeo de Educación Superior (EEES) y la implantación de las nuevas titulaciones de Grado en la Universidad, se han producido cambios en la educación orientados a la reducción del número de horas presenciales y poniendo el énfasis en el aprendizaje del alumno. Es un hecho constatado que los alumnos que acceden a la universidad proceden de diferentes ámbitos, y en muchos casos muestran carencias de formación en las asignaturas básicas, lo que dificulta la posibilidad de que se inicien en la práctica del aprendizaje autónomo. Es necesario proporcionar espacios de aprendizaje en el aula, y también, oportunidades para que el estudiante programe su acción formativa de manera progresiva, en función de sus conocimientos y en base a sus necesidades.

En este contexto las TIC juegan un papel fundamental ya que ofrecen alternativas a la docencia presencial orientadas a la formación de personas con capacidad de adaptarse y de 
sobrevivir en un ambiente cambiante. Se trata de la educación o el aprendizaje líquido (Bauman, 2007) acorde a nuestros tiempos, basado en el uso de tecnologías y adaptando el conocimiento a las personas.

Con el fin de ofrecer a los estudiantes la posibilidad subsanar las deficiencias detectadas en sus conocimientos de materias básicas, la Universitat Politècnia de València lanzó, en el curso académico 2012-13, la iniciativa de ofertar en su plataforma UPV[X] cursos propedéuticos de Química, Física y Matemáticas en formato MOOC (Méndez, 2013), especialmente dirigidos a los alumnos de nuevo ingreso. En el caso de la asignatura de Química, se diseñó el curso "Introducción a la Química” planteado como un curso completo de prerrequisitos, que abarcara todos los conocimientos que el alumno debe tener en su acceso a la universidad (Gómez, 2014).

Tras varias ediciones en UPV[X] y en base al análisis de los resultados respecto al número de alumnos que conseguían finalizar el curso y superar la prueba final, y la retroalimentación proporcionada por los estudiantes en el foro, nos planteamos ofrecer cursos más selectivos presentando la materia del curso "Introducción a la Química" distribuida en cuatro cursos MOOC. La propuesta, que se materializó en octubre de 2014, tiene la finalidad de satisfacer las necesidades formativas de los estudiantes al poder elegir los cursos que consideren más adecuados para conseguir los conocimientos que precisen, en resumen, se trata de una docencia líquida adaptada a cada individuo.

Todos los cursos MOOC ofertados en la plataforma UPV[X] se han diseñado siguiendo el modelo instruccional, en el que la secuenciación de contenidos es fundamental (ZapataRos, 2013a; 2013b; 2014) y debe desarrollarse teniendo en cuenta los siguientes aspectos:

- Definición clara de objetivos.

- Preparación de materiales atractivos que fomenten y sean el soporte de la formación no presencial.

- Diseño de actividades complementarias que permitan la evaluación formativa.

- Prueba de evaluación final sumativa.

En enero de 2015 la UPV acuerda la incorporación de todos los cursos MOOC de UPV[X] a la plataforma edX del MIT (Massachusetts Institute of Technology) y la Universidad de Harward. A partir de este momento los cuatro cursos de Química se ofrecen en edX en formato MOOC y además se ofrecen en la propia UPV, como SPOC, es decir, "Small Private Online Courses”, más personalizados, con retroalimentación instantánea y dirigidos a un público más reducido que los MOOC. A partir del próximo curso académico los estudiantes que accedan a la UPV podrán seguir los SPOC y disponer de todos los materiales en la propia plataforma educativa PoliformaT.

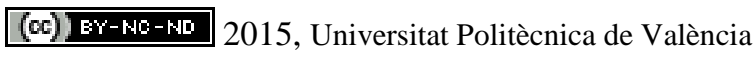

Congreso In-Red (2015) 


\section{Objetivos}

El objetivo general de la comunicación es divulgar la experiencia del uso de la plataforma UPV $[X]$ para dinamizar el aprendizaje líquido de la Química Básica y animar la participación de otros profesores universitarios en este tipo de iniciativas. Para ello nos hemos planteado los siguientes objetivos específicos:

- Explorar la oferta existente de cursos on-line de Química básica.

- Diseñar una serie de cursos orientados a cubrir la posible demanda de formación líquida de la Química básica.

- Realizar un análisis crítico del desarrollo de los mismos.

\section{Desarrollo de la innovación}

Se ha realizado una revisión de la oferta de cursos de Química básica, orientados a cubrir las necesidades de un estudiante de primer curso de química, en las plataformas en español que ofrecen cursos gratis y online, como son la de UNED Abierta, Miríada X y Coursera. Ni la UNED Abierta, ni las 29 universidades que tienen cursos en Coursera ofrecen cursos MOOC en esta línea. Tan sólo Miriada X, en la que participan 51 universidades, oferta el curso "Descubriendo la Química: de la alquimia a las partículas subatómicas", que presenta un recorrido por la historia de la Química, desde los primeros alquimistas hasta los nuevos materiales. Es un curso de la Universitat de Girona. (https://www.miriadax.net/web/descubriendo-quimica-2edicion) que nada tiene que ver con los objetivos de proporcionar formación completa a un estudiante de nuevo ingreso a la Universidad.

En base a la escasa oferta sobre la enseñanza de la Química básica on-line se han diseñado e implementado los cuatro cursos, que se corresponden con los contenidos que los alumnos deben conocer a su entrada a la Universidad. Los cursos son:

- Introducción a la estructura de la materia (MOOC 1)

- El enlace químico y las interacciones moleculares (MOOC 2)

- Formulación y nomenclatura de compuestos químicos (MOOC 3)

- Reacciones químicas y cálculos estequiométricos (MOOC 4)

El diseño de estos cursos se corresponde con las características de un MOOC, donde la adecuada delimitación y secuenciación de los contenidos es esencial. La estructura de cada curso está basada en unidades didácticas, cada unidad se compone de varias lecciones y el contenido se imparte a través de un conjunto articulado de grabaciones en vídeo, actividades de aplicación y de autoevaluación, y de evaluación final. El material también se 
presenta en soporte documental PDF para descargar. El foro es el canal de comunicación que nos ha proporcionado retroalimentación y actividad tutorial.

Se ha realizado un análisis exhaustivo sobre el volumen de trabajo que ha supuesto, para los profesores implicados, la preparación y el seguimiento de los MOOC, así como sobre los alumnos que han participado y los resultados obtenidos.

\section{Resultados}

En la Tabla 1 se recoge para cada uno de los MOOC el material preparado considerando tanto las presentaciones de cada uno de ellos en la plataforma, las grabaciones en vídeo de las lecciones y los ejercicios y el material que lleva implícito un proceso de formación, tanto de las actividades a realizar por el alumno como los exámenes para su evaluación.

Tabla 1. Cantidad de materiales preparados en cada uno de los MOOC y en total

\begin{tabular}{lccccc}
\hline Materiales & MOOC 1 & MOOC 2 & MOOC 3 & MOOC 4 & TOTAL \\
\hline Ficha presentación & 1 & 1 & 1 & 1 & 4 \\
Video presentación & 1 & 1 & 1 & 1 & 4 \\
Unidades Didácticas & 3 & 4 & 10 & 6 & 23 \\
Lecciones & 10 & 15 & 23 & 13 & 61 \\
Videos de lección & 10 & 15 & 23 & 13 & 61 \\
Videos de ejercicios & & 8 & 23 & 8 & 39 \\
Documentos en PDF & 10 & 23 & 46 & 21 & 100 \\
Actividades & 10 & 21 & 46 & 13 & 90 \\
Evaluaciones & 3 & 4 & 10 & 6 & 23 \\
Examen final & 1 & 1 & 1 & 1 & 4 \\
\hline
\end{tabular}

Se ha estimado el tiempo que ha supuesto el diseño del material en PowerPoint para realizar grabación de cada lección, la propia grabación, la preparación de los ejercicios de autoevaluación y los exámes, el trabajo de revisión y supervisión de la tarea del personal técnico asignado a introducir los ejercicios de autoevaluación y los exámenes finales en la plataforma UPV[X], que está preparada para introducir texto pero no las fórmulas químicas, lo que supone supervisar minuciosamente cada una de ellas. También se ha estimado el tiempo dedicado, por término medio, a la supervisión de las transcripciones, que presentan una dificultad considerable habida cuenta de la especificidad del vocabulario de la Química, en especial lo que se refiere a la nomenclatura de los compuestos orgánicos e inorgánicos en el MOOC 3. 
Cabe señalar que el material elaborado debe de ser original y no se puede hacer uso de imágenes si no son de acceso libre, lo que ha supuesto un tiempo añadido el preparar las imágenes y cualquier otro material complementario para ilustrar los contenidos de cada una de las lecciones presentadas.

Considerando el material elaborado, que se recoge en la tabla anterior, y el tiempo medio en la preparación de cada uno de los apartados mencionados se ha realizado una valoración del tiempo empleado que se detalla en la Tabla 2.

Tabla 2. Tiempo medio de dedicación de cada una de las actividades en horas

\begin{tabular}{lccccc}
\hline \multirow{2}{*}{\multicolumn{1}{c}{ Actividades }} & \multicolumn{5}{c}{ Tiempo empleado } \\
\cline { 2 - 6 } & MOOC 1 & MOOC 2 & MOOC 3 & MOOC 4 & TOTAL \\
\hline Preparación diapositivas & 30 & 69 & 138 & 63 & 300 \\
Grabaciones & 5 & 15 & 30 & 11 & 61 \\
Revisión plataforma & 10 & 30 & 60 & 22 & 122 \\
Transcripciones & 5 & 15 & 30 & 11 & 61 \\
Preparación de ejercicios & 9 & 12 & 30 & 40 & 91 \\
de autoevaluación & 2 & 3 & 6 & 6 & 17 \\
Preparación de exámenes & 61 & 144 & 294 & 153 & 652 \\
TOTAL & & & &
\end{tabular}

Hay que hacer notar que en este tiempo de dedicación no se ha tenido en cuenta el empleado en reuniones para el diseño de todas las actividades a realizar, la coordinación de los profesores que participamos y la solución de los problemas técnicos que han ido surgiendo a lo largo del proceso. Por otro lado, cuando los cursos están abiertos los profesores debemos de estar disponibles para contestar las preguntas que se realizan a través del foro lo cual supone una dedicación añadida.

Se han analizado los datos recogidos sobre el número de alumnos que han participado y los que han finalizado cada curso que se muestran en la Tabla 3. Conviene considerar que toda persona que desea obtener información del curso debe estar inscrita, por ello el número de personas que lo están es superior al numero de las que lo que inician y lo siguen.

Se destaca que el curso de "Introducción a la Química” lo han finalizado el 25,4 \% y el $30,2 \%$ de los alumnos inscritos en sus dos últimas ediciones, respectivamente. El curso de “Formulación y nomenclatura de compuestos químicos”, que tiene una duración mayor que los otros tres MOOC ofertados (Tabla1), muestra un porcentaje similar al de "Introducción a la Química” (26,7\%).

Considerando los cursos "Introducción a la estructura de la materia” (59,3\%), "El enlace químico y las interacciones moleculares” (61,5\%) y "Reacciones químicas y cálculos estequiométricos $(60,0 \%)$ observamos que los alumnos que han finalizado casi duplican a los cursos de mayor duración, de lo cual deducimos que la duración del curso condiciona la 
$M^{a}$ Teresa Pardo, Isabel Morera, Juan A. Llorens-Molina, Carmen Gómez e Ignacio Despujol

finalización de este.

Tabla 3. Número y porcentaje de alumnos que han iniciado y terminado los cursos

\begin{tabular}{|c|c|c|c|c|}
\hline \multirow{2}{*}{ Fechas } & \multirow{2}{*}{ Cursos } & \multicolumn{3}{|c|}{ Alumnos } \\
\hline & & Inician & Terminan & $\%$ \\
\hline $02 / 2014$ & Introducción a la Química & 213 & 54 & 25,4 \\
\hline 07/2014 & Introducción a la Química & 222 & 67 & 30,2 \\
\hline $10 / 2014$ & Introducción a la estructura de la materia & 81 & 48 & 59,3 \\
\hline 10/2014 & El enlace químico y las interacciones moleculares & 91 & 56 & 61,5 \\
\hline 10/2014 & Formulación y nomenclatura de compuestos químicos & 180 & 48 & 26,7 \\
\hline $10 / 2014$ & Reacciones químicas y cálculos estequiométricos & 85 & 51 & 60,0 \\
\hline
\end{tabular}

Se ha realizado un análisis de la motivación de los alumnos por realizar los cursos y las respuestas se han agrupado en 8 ítems:

1. Aprender química y ampliar conocimientos en esta materia

2. Satisfacción personal, curiosidad e interés

3. Actualización de conocimientos

4. Objetivos profesionales o académicos

5. Finalidad propedéutica

6. Prestigio de la plataforma y/o universidad que convoca el curso

7. Comparación de contenidos con otros materiales didácticos

8. Lenguaje (español) del curso, que otras plataformas no ofrecen

En las figuras 1-4 se muestran los porcentajes de cada uno de los ítems para los diferentes MOOC.

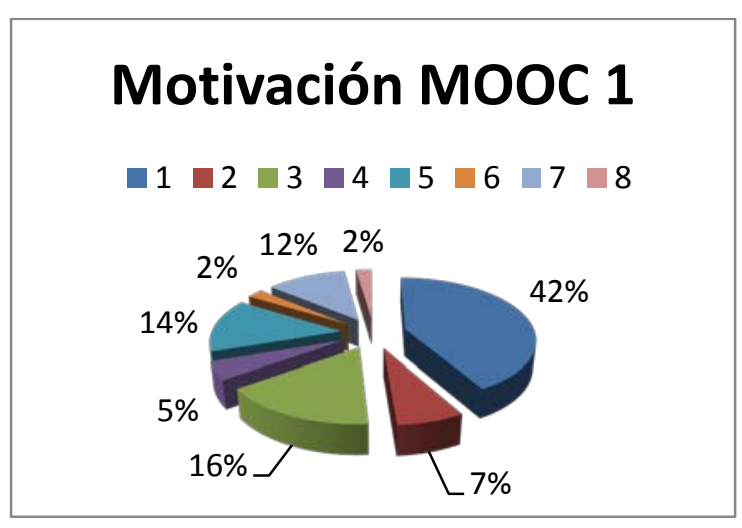

Fig. 1 Porcentajes de motivación en el MOOC 1

(cc)) EY-NC-ND 2015, Universitat Politècnica de València

Congreso In-Red (2015) 


\section{Motivación MOOC 2}

$\square 1 \square 2 \square 3 \square 4 \square 5 \square 6 \square 7 \square 8$

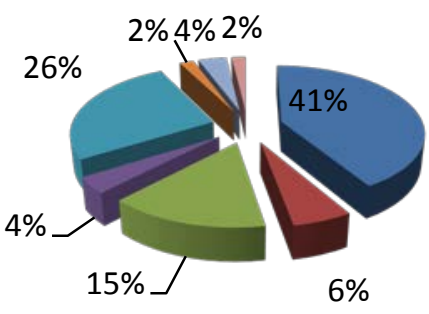

Fig. 2 Porcentajes de motivación en el MOOC 2

\section{Motivación MOOC 3}
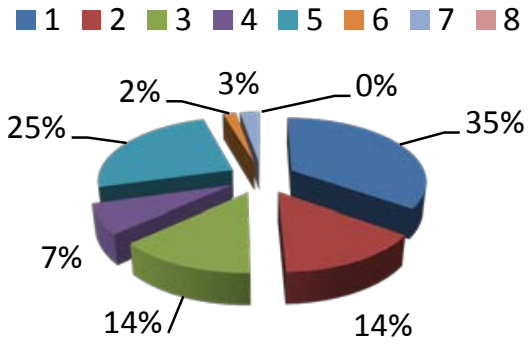

Fig. 3 Porcentajes de motivación en el MOOC 3

\section{Motivación MOOC 4}
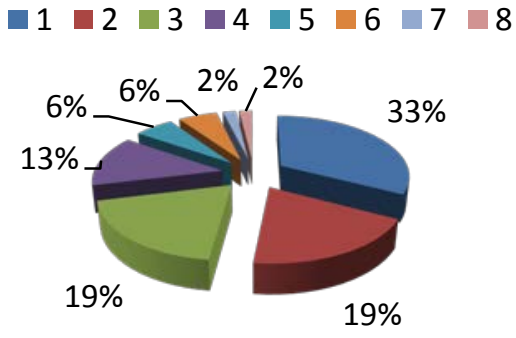

Fig. 4 Porcentajes de motivación en el MOOC 4 
En cuanto a la motivación se observa claramente que destaca el interés de los alumnos por aprender y ampliar conocimientos en la materia (ítem 1). El resto de las preferencias de alumnado se pueden agrupar en dos perfiles:

- Interés genérico basado en la satisfacción personal y la curiosidad (ítems 2 y 3 )

- Objetivos académicos o de desarrollo profesional (ítems 4 y 5)

Cabe destacar también que el ítem 7, “Comparación de contenidos con otros materiales didácticos" alcanza una proporción mayor en el curso "Introducción a la estructura de la materia”.

\section{Conclusiones}

En la docencia on-line la selección, la organización, la presentación y la secuenciación de contenidos es fundamental, así como y la duración de los vídeos y del curso.

El contenido de los cursos debe ser autosuficiente para permitir la utilización autónoma del material por el estudiante, adaptando la secuencia de aprendizaje a sus necesidades y conocimientos previos.

Publicitar el curso y proporcionar información sobre el contenido y desarrollo del mismo condiciona su seguimiento y evita que el estudiante se cree falsas expectativas.

La participación en los foros nos permite obtener retroalimentación para mejorar el diseño de los cursos.

Los datos recogidos en encuestas sobre las opiniones de los alumnos respecto al grado de satisfacción y de utilidad indican que, en general, se cubren sus expectativas.

El grado de seguimiento y finalización de los cursos, por temino medio, se ha incrementado considerablemente al adecuar el contenido al tiempo de duración del curso.

En general, el seguimiento de los cursos viene motivado por el interés de adquirir conocimientos en la disciplina de Química

El trabajo realizado y el tiempo invertido por el profesorado y los técnicos ha sido mucho, pero ha sido una experiencia muy satisfactoria y compensada por el éxito y resultado obtenido.

La mejora en cuanto al diseño de estos cursos, debería orientarse hacia la incorporación de actividades de aprendizaje y evaluación con nuevos formatos, más allá de las pruebas objetivas de opción múltiple, para lo que se requeriría adecuar la plataforma. 
Del MOOC al SPOC.Una experiencia para el aprendizaje líquido de la Química básica

\section{Referencias}

BAUMAN, Z. (2007). Los retos de la educación en la modernidad líquida. Barcelona: Gedisa.

GÓMEZ, C et al. (2014). "Diseño y producción de cursos MOOC para el aprendizaje de Química Básica”. En INRED 2014. Valencia : UPV. p. 88-101.

Méndez, C. M. (2013). "Diseño e implementación de cursos abiertos masivos en línea (MOOC): expectativas y consideraciones prácticas”. En Revista de la Educación a Distancia, 39. http://www.um.es/ead/red/39 [Consultado: 25 de mayo de 2015]

Zapata-Ros, M. (2013a). "MOOCs, una visión crítica y una alternativa complementaria: La individualización del aprendizaje y de la ayuda pedagógica”. En Campus Virtuales, II, (1), p. 20-38.

Zapata-Ros, M. (2013b). Las teorías del aprendizaje y el diseño instruccional. El esquema incompleto. http://redesabiertas.blogspot.com.es/2013/04/las-teorias-del-aprendizaje-y-eldiseno.html [Consultado: 20 de mayo de 2015]

ZAPATA-ROS, M. (2014). Los MOOC en la crisis de la Educación Universitaria.: Docencia, diseño y aprendizaje. 